Irish Math. Soc. Bulletin

Number 83, Summer 2019, 5-7

ISSN 0791-5578

\title{
Generalization of an Identity about Euler's totient function
}

\author{
MEI BAI, WENCHANG CHU AND NADIA N. LI
}

\begin{abstract}
A general formula about arithmetic functions is established that contains some interesting identities as special cases, including one about Euler's totient function proposed by Max A. Alekseyev (2011).
\end{abstract}

In number theory, Euler's totient function $\varphi(n)$ is well-known, which counts the natural numbers $\leq n$ that are relatively prime to $n$ and satisfies the property

$$
\sum_{d \mid n} \varphi(d)=n
$$

Max A. Alekseyev [1] proposed the following monthly problem. For a positive integer $m$, prove that

$$
\sum_{k=0}^{m-1} \varphi(2 k+1)\left\lfloor\frac{m+k}{2 k+1}\right\rfloor=m^{2}
$$

where $\lfloor x\rfloor$ denotes the integer part for a real number $x$.

Inspired by this problem, we shall establish the following general formula.

Theorem 1 (Main theorem). Let $F(n)=\sum_{d \mid n} f(d)$, then

$$
\sum_{n=1}^{m} F(2 n-1)=\sum_{k=1}^{m} f(2 k-1)\left\lfloor\frac{m+k-1}{2 k-1}\right\rfloor .
$$

Proof. By replacing $d$ by $2 k-1$ and then interchanging the summation order, we have

$$
\sum_{n=1}^{m} F(2 n-1)=\sum_{n=1}^{m} \sum_{d \mid 2 n-1} f(d)
$$

2010 Mathematics Subject Classification. Primary 11A25, Secondary 05A19.

Key words and phrases. Euler's totient function; Divisor function; Liouville's $\lambda$ function. Received on 4-4-2019.

During this work, the first author was partially supported by the Science foundation of Zhoukou Normal University (ZKNUB3201701), and the third author by the National Science foundation of China (Youth Grant No.11601543). 


$$
\begin{aligned}
& =\sum_{n=1}^{m} \sum_{(2 k-1) \mid(2 n-1)} f(2 k-1) \\
& =\sum_{k=1}^{2 m-1} f(2 k-1) \sum_{\substack{n=1 \\
(2 k-1) \mid(2 n-1)}}^{m} 1 .
\end{aligned}
$$

The last sum counts the number of odd multiples of $2 k-1$ from 1 to $2 m-1$, which is equal to the number of all the multiples of $2 k-1$ minus that of the even multiples of $2 k-1$. Hence we have

$$
\sum_{\substack{n=1 \\(2 k-1) \mid(2 n-1)}}^{m} 1=\left\lfloor\frac{2 m-1}{2 k-1}\right\rfloor-\left\lfloor\frac{2 m-1}{4 k-2}\right\rfloor=\left\lfloor\frac{m+k-1}{2 k-1}\right\rfloor .
$$

This proves the formula displayed in Theorem 1.

Replacing $f$ by $\varphi$ in Theorem 1, we confirm immediately Alekseyev's identity (1).

Instead, by assigning, in Theorem $1, F$ to be $\sigma_{\ell}$, the $\ell$ th power sum of the divisors of $n$

$$
\sigma_{\ell}(n)=\sum_{d \mid n} d^{\ell}
$$

we derive another interesting formula:

\section{Corollary 2.}

$$
\sum_{k=1}^{m} \sigma_{\ell}(2 n-1)=\sum_{k=1}^{m}(2 k-1)^{\ell}\left\lfloor\frac{m+k-1}{2 k-1}\right\rfloor .
$$

Furthermore, we can work out an identity for the Liouville function, which is defined by

$$
\lambda(n)=(-1)^{k_{1}+k_{2}+\cdots+k_{m}}
$$

if $n$ is factorized into $n=\prod_{i=1}^{m} p_{i}^{k_{i}}$ with $\left\{p_{i}\right\}_{i=1}^{m}$ being distinct primes. The $\lambda$ function admits the property

$$
\sum_{d \mid n} \lambda(d)= \begin{cases}1, & \text { if } n \text { is a square } \\ 0, & \text { otherwise. }\end{cases}
$$

Now take $f(n)=\lambda(n)$ in Theorem 1 . We can see that $\sum_{n=1}^{m} F(2 n-1)$ is, in fact, the number $\lfloor\sqrt{2 m}\rfloor$ of odd squares from 1 to $2 m$ minus the number $\left\lfloor\sqrt{\frac{m}{2}}\right\rfloor$ of even squares from 1 to $2 m$ :

$$
\sum_{n=1}^{m} F(2 n-1)=\lfloor\sqrt{2 m}\rfloor-\left\lfloor\sqrt{\frac{m}{2}}\right\rfloor=\left\lfloor\sqrt{\frac{m}{2}}+\frac{1}{2}\right\rfloor .
$$

We have consequently established the following identity.

\section{Corollary 3.}

$$
\sum_{k=1}^{m} \lambda(2 k-1)\left\lfloor\frac{m+k-1}{2 k-1}\right\rfloor=\left\lfloor\sqrt{\frac{m}{2}}+\frac{1}{2}\right\rfloor .
$$


There exist similar results in the literature. In fact, one can find in Burton [2, Theorem 6.11] the following formula. Suppose that

$$
F(n)=\sum_{d \mid n} f(d)
$$

then there holds

$$
\sum_{n=1}^{m} F(n)=\sum_{k=1}^{m} f(k)\left\lfloor\frac{m}{k}\right\rfloor .
$$

Analogously, we can deduce from (2) the following identities:

$$
\begin{aligned}
& \sum_{k=1}^{m} \varphi(k)\left\lfloor\frac{m}{k}\right\rfloor=\left(\begin{array}{c}
m+1 \\
2
\end{array}\right), \\
& \sum_{n=1}^{m} \sigma_{\ell}(n)=\sum_{k=1}^{m} k^{\ell}\left\lfloor\frac{m}{k}\right\rfloor, \\
& \sum_{n=1}^{m} \lambda(n)\left\lfloor\frac{m}{n}\right\rfloor=\lfloor\sqrt{m}\rfloor .
\end{aligned}
$$

Among these formulae, (3) and the two particular cases $\ell=0,1$ of (4) appeared previously in Burton's book (Page 120, Corollaries $1 \& 2$ ).

\section{REFERENCES}

[1] M. A. Alekseyev, Squares from totients: Problem 11544, Amer. Math. Monthly 118 (2011), Page 84; Solution ibid 119 (2012), Page 807.

[2] David M. Burton, Elementary Number Theory (6th edition), McGraw Hill Higher Education, 2007.

Mei Bai received her Masters degree at Zhengzhou University (P. R. China). She is currently a lecturer and teaches real analysis at Zhoukou Normal University (Henan, P. R. China).

Wenchang Chu is at present a professor at the University of Salento (Lecce, Italy). His research interests cover combinatorial analysis and special functions, highlighted by extensive publications in both classical hypergeometric series and $q$-series.

Nadia Na Li received her Ph.D degree at Dalian University of Technology (P. R. China). She is currently associate professor and teaches real analysis at Zhoukou Normal University (Henan, P. R. China) .

M. Bai and N. N. li: School of Mathematics and Statistics Zhoukou Normal University, Zhoukou (Henan), P. R. China

W. Chu: Department of Mathematics and Physics

University of SAlEnto, P. O. Box 193

73100 LECCE, ITALY

E-mail address: baimei0418@163.com, chu.wenchang@unisalento.it and lina2017@sohu.com 